\title{
Spacing Optimization for Active Droplet Sorting in Microfluidic Networks Using Genetic Algorithm
}

\author{
E.M. Arun Sankar, Mohammad Shahab and Raghunathan Rengaswamy* \\ Department of Chemical Engineering, Indian Institute of Technology Madras, Chennai 600036, \\ India \\ *email: raghur@iitm.ac.in
}

Supporting Information

$\underline{\text { Video1 and Video } 2}$

Drops were sent at two different inlet spacings to the network. Video 1 shows the simulated movement of drops through the network for one inter-droplet spacing and Video 2 shows the simulated movement of same drops for another inter-droplet spacing at the inlet. Except inlet spacing all other parameters were the same for the two simulations. The routes chosen by the drops in the network are different for the two cases. In Video $1,1^{\text {st }}$ and $3^{\text {rd }}$ drops exit through the Sink 1 and $2^{\text {nd }}$ and $4^{\text {th }}$ drops exit through Sink 2 . In Video $2,1^{\text {st }}$ and $2^{\text {nd }}$ drops exit through Sink 2 and $3^{\text {rd }}$ and $4^{\text {th }}$ drops leave through Sink 1.

Video 3, Video 4 and Video 5

Video 3, Video 4 and Video 5 show the effect of the upper bound, $d^{U}$ for the inlet spacing. The sequence $\mathrm{AAAB}$ is sent to the network at an inlet spacing identified using Genetic Algorithm. The drops are labelled in the order of entry to the network. '1' -drop A, '2' -drop A, '3' -drop A, '4' drop B. Video 3 shows the movement of drops at a spacing that resulted highest fitness ( fitness = 1) for $d^{U}=20000 \mu \mathrm{m}$. All the drops A leave through the Sink 1 and drop B leaves through Sink 2. Video 4 shows the movement of drops at a spacing that resulted best fitness (fitness $=0.67$ ) for $d^{U}=10000 \mu \mathrm{m}$. Here one drop A and the drop B leave through Sink 1 and two drops A leave through Sink 2. Video 5 shows the movement of drops at a spacing that resulted best fitness (fitness $=0.33$ ) for $d^{U}=2000 \mu \mathrm{m}$. Here two drops A and the drop B leave through Sink 1 and one drop A leaves through Sink 2. With a large $d^{U}$ (as in the case of $d^{U}=20000 \mu \mathrm{m}$ ), a drop has a choice to wait till its leading drop covers a portion of the network significant enough to take the favorable decision.

Reduction of equation (13) to equation (19)

It is convenient to look the RHS of equation (13) as the linear combination of the columns of $\operatorname{inv}(G)$. Let $H=i n v(G)$. $H$ is a square matrix of dimension $2 n+b$. As we have considered a $3 \mathrm{x}$ 3 grid network, $\mathrm{H}$ has 54 rows and columns. From equation (13), one can simply note that the 
pressure at each node and flowrate in each branch are linearly related to the inlet flow to the network and pressures at the sink nodes. Equation (13) given in the manuscript for the considered network is given below:

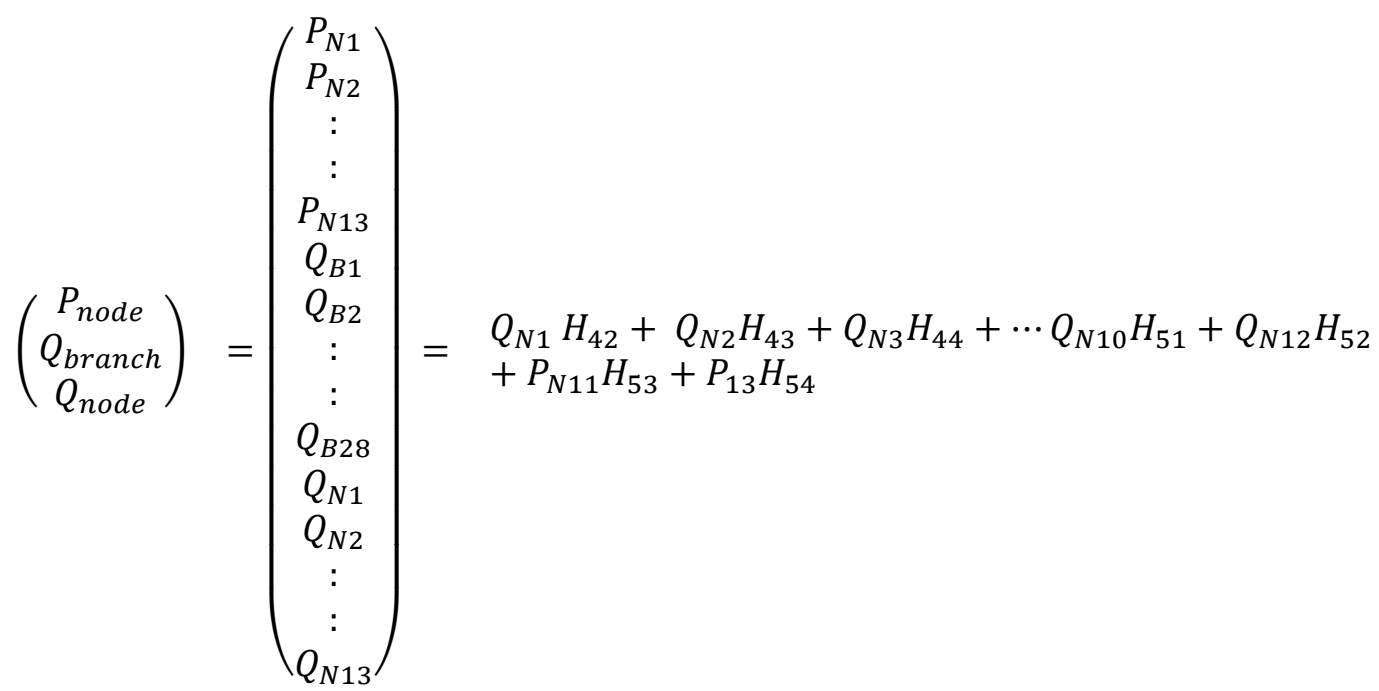

Here $H_{i}$ denotes $i^{\text {th }}$ column of $H(i=1,2, . ., 54), Q_{N j}$ and $P_{N j}$ represents net flowrate and pressure at node $j(j=1,2, . ., 13)$ and $Q_{B l}$ represents the flowrate in branch $l(l=1,2, . ., 28)$ (Refer Figure 3 for the numbering of nodes and branches). As evident from the RHS of equation (13), the first 41 columns of $H$ are multiplied with 0s. The pressure at each node and flowrates in each branch are linear combinations of the $42^{\text {nd }}$ to $54^{\text {th }}$ columns of $H$. Interestingly, the elements in $H_{53}$ and $H_{54}$ corresponding to the $Q_{B 1}$ to $Q_{B 28}$ are equal in magnitude with opposite signs. This is true for any network and for any choice of the sink nodes. Therefore, if the applied pressures at the two sink nodes are equal, then elements corresponding to $Q_{B 1}$ to $Q_{B 28}$ in the last two terms on the RHS of the above equation will add to 0 . We already know that except $Q_{N 2}, Q_{N 11}$ and $Q_{N 13}$, the net flow rates at all other nodes are 0 . Therefore, the flowrates in each branch is linearly proportional to the inlet flowrate, $Q_{\text {in }}\left(=Q_{N 2}\right)$ with 0 intercept as given below:

$$
\left(\begin{array}{c}
P_{\text {node }} \\
Q_{\text {branch }} \\
Q_{\text {node }}
\end{array}\right)=\left(\begin{array}{c}
P_{N 1} \\
P_{N 2} \\
\vdots \\
: \\
P_{N 13} \\
Q_{B 1} \\
Q_{B 2} \\
\vdots \\
: \\
Q_{B 28} \\
Q_{N 1} \\
Q_{N 2} \\
: \\
: \\
Q_{N 13}
\end{array}\right)=Q_{N 2} H_{43}
$$

